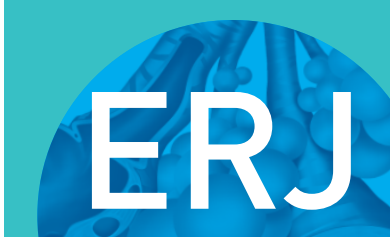

open research

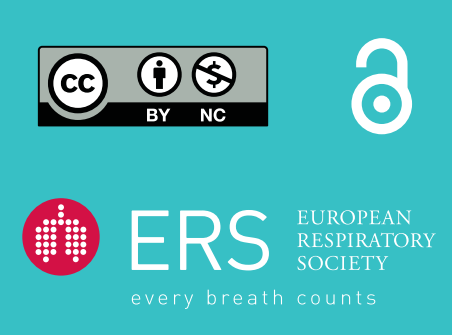

\section{Analysis of pathological changes in the epithelium in COVID-19 patient airways}

\section{To the Editor:}

The outbreak of severe acute respiratory syndrome coronavirus 2 (SARS-CoV-2) has caused more than 70 million infections and approximately 1.6 million deaths worldwide [1]. However, the pathological changes in coronavirus disease 2019 (COVID-19) airways remain unclear due to limited accessibility of autopsy or biopsy.

Here, we report one of the first pathological changes to the epithelium in the severe COVID-19 patient airways. The COVID-19 lungs exhibited much MUC5AC-containing mucus accumulation in many bronchioles. The number of mucus-producing goblet cells was dramatically increased, and the number of multiciliated cells and bronchiolar club cells was significantly reduced. Interleukin (IL)-13 levels were also dramatically increased in the COVID-19 lungs. The levels of secretoglobin CC16 secreted by club cells were significantly reduced in nine COVID-19 patients. Furthermore, SARS-CoV-2 nucleocapsid proteins were detected in human airway epithelial cells after in vitro infection.

A 66-year-old man from Shenzhen, Guangdong Province, China, travelled to Wuhan on 29 December 2019 and returned to Shenzhen on 04 January 2020. He was admitted to the hospital on 11 January 2020 with clinical symptoms of cough, fever, myalgia and mild dyspnoea. He was a non-smoker with the comorbidity of hypertension. SARS-CoV-2 infection was identified by performing a real-time reverse transcriptase (RT)-PCR assay on a nasopharyngeal swab specimen [2]. Noninvasive ventilation and mechanical ventilation were administered to him on 11 January 2020 and 20 January 2020, respectively. After admission to the intensive care unit (ICU) on 25 January 2020, he developed respiratory failure, received lung transplantation therapy on 25 February 2020, and was declared clinically dead on 26 February 2020. On chest radiographs, we observed multiple patchy shadows with a progressive increase in the area of lung from 12 January to 22 January (figure 1a-c). Chest computed tomography (CT) showed bilateral and peripheral ground-glass opacities associated with intralobular septal thickening in his lungs on 13 January (figure 1d, e). Lesions in his lungs increased in extent with obvious heterogeneous density and air bronchogram on 23 January (figure 1f, g). On 30 January, his lungs exhibited a dramatic decrease in brightness with much more patchy shadows (figure 1h). From 01 February, the brightness of his lungs was further decreased with a large area of patchy shadow (figure 1i, j). Samples from the injured lungs of this patient were embedded in paraffin and sectioned for examination.

To test for pathological changes in COVID-19 airways, we analysed lung sections using histological staining. The COVID-19 patient lungs exhibited severe haemorrhage in the airways and alveoli (figure 1k). Many of the COVID-19 distal bronchioles were plugged by mucus, accompanied by goblet cell hyperplasia in the airway epithelium (figure 11). To examine cellular changes in COVID-19 airways, we performed a detailed analysis of the airway epithelium in healthy controls and the patient by immunostaining [3]. We observed that many of the distal bronchioles including the smaller ones in the COVID-19 patient were plugged by MUC5AC-containing mucus (figure $1 \mathrm{~m}, \mathrm{n}, \mathrm{p}, \mathrm{q}$ ). $\mathrm{MUC}^{\mathrm{A}} \mathrm{AC}^{+}$goblet cells mainly localise in bronchi and are barely detectable in distal bronchioles in humans [4]. By contrast, a large number of MUC5 $\mathrm{AC}^{+}$goblet cells were observed in more than half of the bronchioles including the smaller ones in the COVID-19 patient compared with healthy controls (figure $1 \mathrm{~m}, \mathrm{o}, \mathrm{p}, \mathrm{r}$ ). A quantitative reverse

@ERSpublications

Severe COVID-19 patient airways plugged by MUC5AC-containing mucus exhibit hyperplasia of goblet cells, and hypoplasia of multiciliated cells and club cells, as well as significantly reduced CC16 and MUC5B levels, and increased IL-13 levels https://bit.ly/2M2NcdO

Cite this article as: Yin W, Cao W, Zhou G, et al. Analysis of pathological changes in the epithelium in COVID-19 patient airways. ERJ Open Res 2021; 7: 00690-2020 [https://doi.org/ 10.1183/23120541.00690-2020].

Copyright $\odot$ The authors 2021. This version is distributed under the terms of the Creative Commons Attribution

Non-Commercial Licence 4.0. For commercial reproduction rights and permissions contact permissions@ersnet.org 

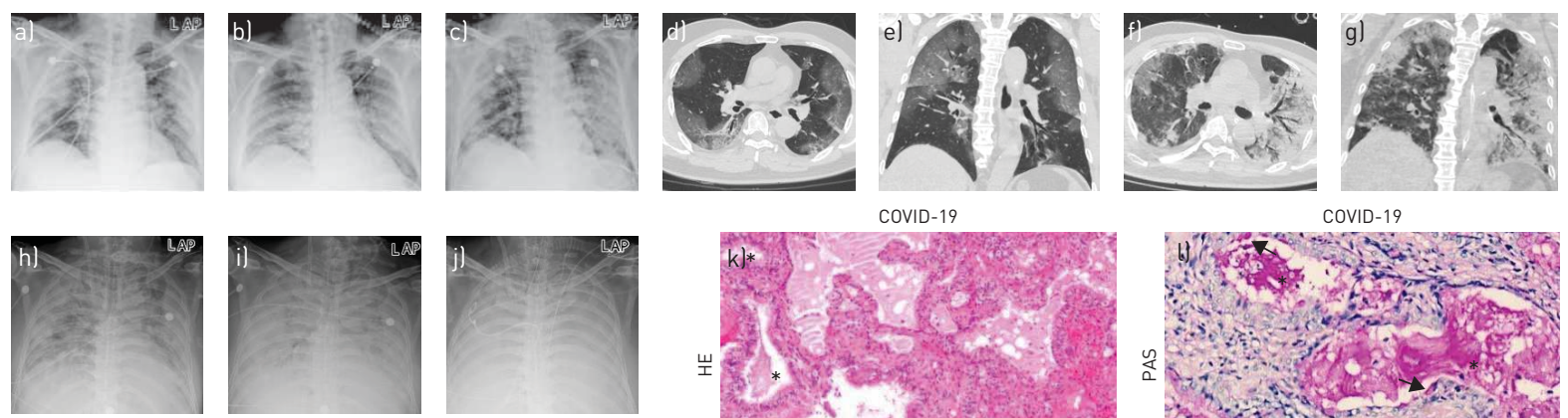

\section{COVID-19}
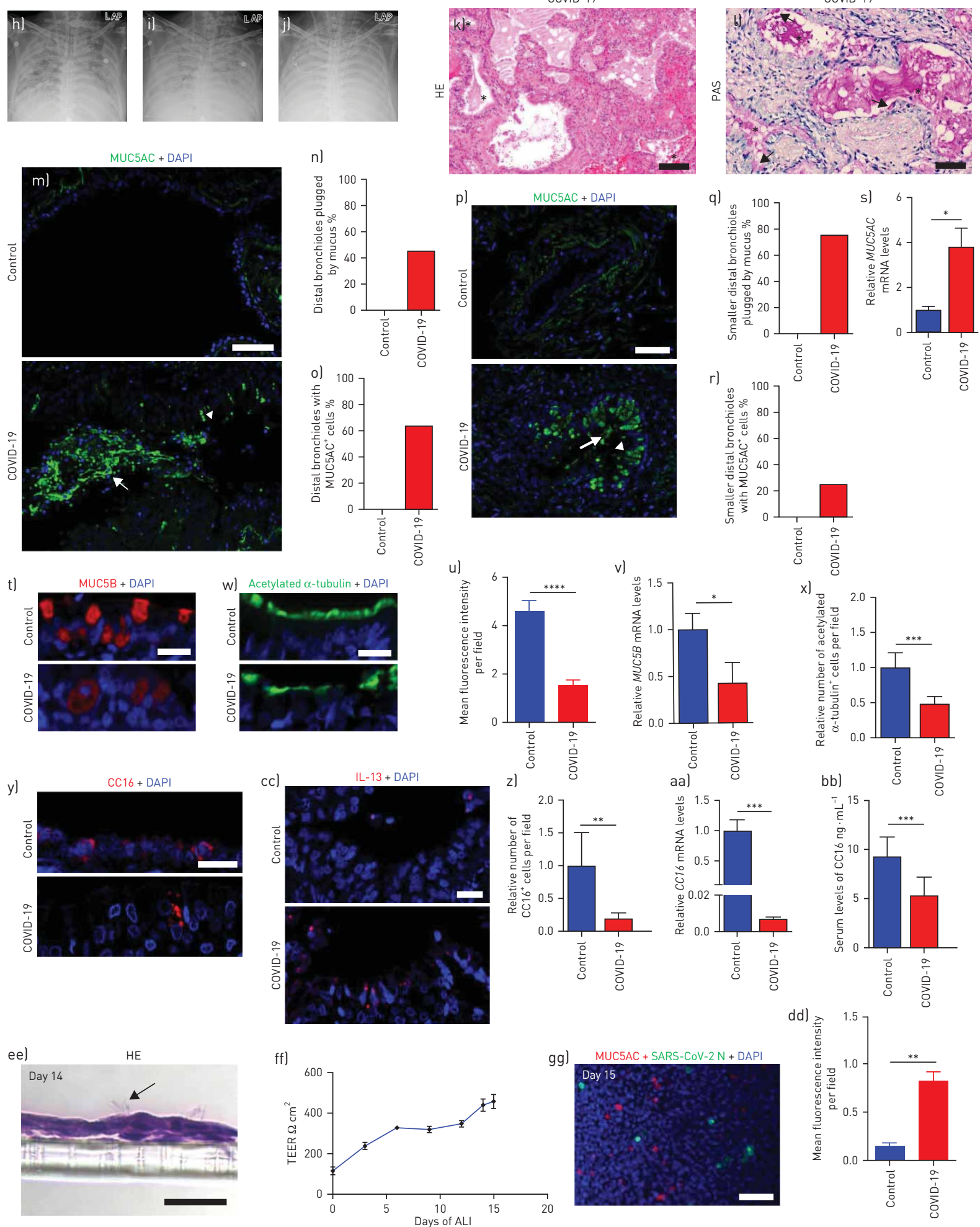

FIGURE 1 Coronavirus disease 2019 (COVID-19) patient lungs exhibit mucus plugs and pathological changes in the airway epithelium. Chest radiographs obtained on a) 12 January 2020, b) 17 January 2020 and c) 22 January 2020. d, e) Representative lung CT image on 13 January 2020. f, g) Representative transverse lung CT image on 23 January 2020. Chest radiographs obtained on h) 30 January 2020 i) 1 February 2020 and 
j) 15 February 2020. k) Representative images of lung tissue sections from the COVID-19 patient stained with haematoxylin and eosin (HE). Asterisks indicate airways. () Representative images of lung tissue sections from the COVID-19 patient stained with periodic acid-Schiff (PAS). The arrows point to goblet cells. Asterisks indicate mucus plugs in the airways. m) Immunostaining for MUC5AC (green) and DAPI staining (blue) of sections of larger distal bronchioles from healthy controls $(n=3)$ and the COVID-19 patient. The arrow points to the mucus plug. The arrowhead points to the goblet cell. n) Quantification of the number of larger distal bronchioles plugged by mucus in healthy controls ( $n=3$ ) and the COVID-19 patient. o) Quantification of the number of larger distal bronchioles with MUC5AC ${ }^{+}$cells in healthy controls (n=3) and the COVID-19 patient. p) Immunostaining for MUC5AC (green) and DAPI staining (blue) of sections of the smaller distal bronchioles from healthy controls ( $n=3$ ) and the COVID-19 patient. The arrow points to the mucus plug. The arrowhead points to the goblet cell. q) Quantification of the number of smaller distal bronchioles plugged by mucus in healthy controls ( $n=3$ ) and the COVID-19 patient. r) Quantification of the number of smaller distal bronchioles with MCU5AC $^{+}$cells in healthy controls $(\mathrm{n}=3$ ) and the COVID-19 patient. s) Relative MUC5AC mRNA levels in the lungs from healthy controls and the COVID-19 patient. t) Immunostaining for MUC5B (red) and DAPI staining (blue) of sections of the airways from healthy controls ( $n=3$ ) and the COVID-19 patient. u) Quantification of mean fluorescence intensity of MUC5B immunostaining in the airways in healthy controls (n=3) and the COVID-19 patient. v) Relative MUC5B mRNA levels in the lungs from healthy controls and the COVID-19 patient. w) Immunostaining for acetylated $\alpha$-tubulin (green) and DAPI staining (blue) of sections of small bronchioles from healthy controls $(n=3)$ and the COVID-19 patient. x) Quantification of the relative number of acetylated $\alpha$-tubulin ${ }^{+}$ multiciliated cells in small bronchioles in healthy controls $(n=3)$ and the COVID-19 patient. y) Immunostaining for CC16 (red) and DAPI staining (blue) of sections of distal bronchioles from healthy controls ( $n=3$ ) and the COVID-19 patient. z) Quantification of the relative number of CC16 ${ }^{+}$cells in distal bronchioles in healthy controls $(n=3)$ and the COVID-19 patient. aa) Relative CC16 mRNA levels in the lungs from healthy controls and the COVID-19 patient. bb) Quantification of CC16 levels in serum from healthy controls (n=7) and severe and critical COVID-19 patients (n=9). cc) Immunostaining for interleukin (IL)-13 (red) and DAPI staining (blue) of sections of the lungs from healthy controls (n=3) and the COVID-19 patient. dd) Quantification of mean fluorescence intensity of IL-13 immunostaining in the lungs in healthy controls ( $n=3$ ) and the COVID-19 patient. ee) Representative images of cross sections of air-liquid interface (ALI) cultures stained with HE. The arrow points to cilia. ff) Trans-epithelial electrical resistance (TEER) measurements. gg) Immunostaining for MUC5AC (red), severe acute respiratory syndrome coronavirus 2 nucleocapsid proteins (SARS-CoV-2 N; green) and DAPI staining (blue) in human bronchial epithelial cells at the ALI after a 14-day differentiation. Scale bars: $100 \mu \mathrm{m}$ ( $k, \mathrm{l}, \mathrm{m}, \mathrm{p}, \mathrm{gg}$ ), $20 \mu \mathrm{m}$ (t, $w, y, c c, e e) . *$ : $p<0.05 ;{ }^{* *}: p<0.01 ;{ }^{* * *}: p<0.001 ;{ }^{* * *}: p<0.0001 ;$ unpaired t-test, mean \pm SD.

transcription PCR assay demonstrated MUC5AC mRNA levels were dramatically increased in the COVID-19 lungs compared with healthy controls (figure 1s). MUC5B is required for mucociliary clearance in the airways [5]. Notably, MUC5B protein and mRNA levels appeared to be dramatically reduced in the airway epithelium in the COVID-19 patient compared with healthy controls (fig. 1t-v). Multiciliated cells beat to constantly propel mucus from the distal to proximal airways for clearance of mucus-trapped pathogens and particles [6]. The number of acetylated $\alpha$-tubulin ${ }^{+}$multiciliated cells appeared to be significantly reduced in the small bronchioles in the COVID-19 patient (figure 1w, $\mathrm{x}$ ).

Club cells secrete CC16, a secretoglobin that can attenuate airway mucus production and hypersecretion [7-9]. We observed that the number of $\mathrm{CC} 6^{+}$club cells was significantly reduced in distal bronchioles in the COVID-19 patient compared with healthy controls (figure 1y, z). CC16 mRNA levels appeared to be dramatically reduced in the lungs of the COVID-19 patient (figure 1aa). Next, we examined circulating CC16 levels in serum from seven healthy nonsmoking controls, aged 38-67 years, and nine severe COVID-19 patients, aged 41-72 years including four smokers, by ELISA. CC16 levels were significantly decreased in COVID-19 patients compared with healthy controls (figure 1bb). In humans, IL-13 can induce mucus production in the lungs $[10,11]$. IL-13 levels appeared to be dramatically increased in COVID-19 patients compared with healthy controls (figure 1cc, dd).

To investigate whether human airway epithelial cells are susceptible to SARS-CoV-2, we used primary bronchial epithelial cells cultured in vitro at the air-liquid interface (ALI) as a model of human airway epithelium formation [12]. After a 14-day differentiation (figure 1ee, ff), we performed a 24-h virus inoculation into human airway epithelium at a multiplicity of infection (MOI) of 0.2 , SARS-CoV-2 nucleocapsid proteins were detected in human airway epithelial cells by immunostaining (figure 1gg), indicating that the human airway epithelium is a potential site for SARS-CoV-2 infection.

This study provides one of the first descriptions of the pathological changes in the COVID-19 airway epithelium. The COVID-19 airways are plugged by abundant MUC5AC-containing mucus, accompanied by hyperplasia of goblet cells as well as hypoplasia of club cells and multiciliated cells. It is possible that SARS-CoV-2 infection causes impaired airway epithelial cell homeostasis, leading to mucus overproduction and/or hypersecretion and/or accumulation, ultimately resulting in bronchiole obstruction, dyspnoea or even death. These findings warn that COVID-19 patients with dyspnoea and mucus plugs in the airways should be treated with caution. It is possible that the forced airflow from mechanical ventilation pushes mucus into the alveoli, which may dramatically reduce the air-exchange surface in the lungs and result in more severe hypoxia. Thus, we propose that mucus in the airways should be examined and removed before using mechanical ventilation for COVID-19 patients.

Decreased CC16 levels have been shown to be associated with pulmonary diseases with mucus overproduction, including COPD [13,14] and asthma [15]. CC16 could inhibit mucus production and hypersecretion in mouse airways and the ALI differentiation model of human bronchial epithelial cells [7-9]. IL-13 promotes mucus production in the lungs [10, 11]. Our findings that severe COVID-19 patients exhibit deceased CC16 levels and increased IL-13 levels, reveal other possible causes of airway mucus accumulation in COVID-19 patients. 
Wenguang Yin ${ }^{1,4}$, Weitao Cao ${ }^{1,4}$, Guangde Zhou, ${ }^{2,4}$, Lifei Wang ${ }^{3,4}$, Jing Sun ${ }^{1,4}$, Airu Zhu ${ }^{1}$, Zhongfang Wang ${ }^{1}$, Yumin Zhou ${ }^{1}$, Xiaoqing Liu ${ }^{1}$, Yimin Li $^{1}$, Nanshan Zhong ${ }^{1}$, Jincun Zhao ${ }^{1}$, Lei Liu ${ }^{2}$ and Pixin Ran 1

${ }^{1}$ State Key Laboratory of Respiratory Disease, National Clinical Research Center for Respiratory Disease, Guangzhou Institute of Respiratory Health, the First Affiliated Hospital of Guangzhou Medical University, Guangzhou, China. ${ }^{2}$ Shenzhen Key Laboratory of Pathogen and Immunity, National Clinical Research Center for Infectious Disease, State Key Discipline of Infectious Disease, Shenzhen Third People's Hospital, Second Hospital Affiliated to Southern University of Science and Technology, Shenzhen, China. ${ }^{3}$ Dept of Radiology, National Clinical Research Center for Infectious Disease, Third People's Hospital, Second Hospital Affiliated to Southern University of Science and Technology, Shenzhen, China. ${ }^{4}$ These authors contributed equally.

Correspondence: Pixin Ran, State Key Laboratory of Respiratory Disease, National Clinical Research Center for Respiratory Disease, Guangzhou Institute of Respiratory Health, the First Affiliated Hospital of Guangzhou Medical University, Yanjiang Rd 195, Guangzhou, Guangdong 510182, China. E-mail: pxran@ gzhmu.edu.cn

Received: 16 Nov 2020 | Accepted: 5 Jan 2021

Acknowledgements: We thank Ruiting Sun (State Key Laboratory of Respiratory Disease, National Clinical Research Center for Respiratory Disease, Guangzhou Institute of Respiratory Health, the First Affiliated Hospital of Guangzhou Medical University, Guangzhou, China) for imaging assistance.

Ethics approval: This study was approved by the Ethics Committee of the First Affiliated Hospital of Guangzhou Medical University (number 2020-51). Because of the urgent need to collect data on this emerging infectious disease, the requirement for written informed consent was waived.

Author contributions: W. Yin and P. Ran conceived the project, designed experiments and analysed data; W. Cao L. Liu, G. Zhou, L. Wang and J. Sun contributed to experiments and data analysis; W. Yin, Z. Wang, Y. Zhou, N. Zhong and J. Zhao contributed to discussion and data analysis; A. Zhu, X. Liu and Y. Li contributed to patient recruitment and sample collection. W. Yin and P. Ran wrote the manuscript.

Conflict of interest: None declared.

Support statement: This study was supported by Guangdong Key Research and Development Project grant 2020B1111330001. Funding information for this article has been deposited with the Crossref Funder Registry.

\section{References}

1 World Health Organization. Weekly epidemiological update - 15 December 2020. www.who.int/publications/m/ item/weekly-epidemiological-update-15-december-2020 Date last updated: 15 December 2020.

2 Wang D, Hu B, Hu C, et al. Clinical characteristics of 138 hospitalized patients with 2019 novel coronavirus-infected pneumonia in Wuhan, China. JAMA 2020; 323: 1061-1069.

3 Yin W, Kim HT, Wang S, et al. The potassium channel KCNJ13 is essential for smooth muscle cytoskeletal organization during mouse tracheal tubulogenesis. Nat Commun 2018; 9: 2815.

4 Okuda K, Chen G, Subramani DB, et al. Localization of secretory mucins MUC5AC and MUC5B in normal/ healthy human airways. Am J Respir Crit Care Med 2019; 199: 715-727.

5 Roy MG, Livraghi-Butrico A, Fletcher AA, et al. Muc5b is required for airway defence. Nature 2014; 505: 412-416.

6 Bustamante-Marin XM, Ostrowski LE. Cilia and mucociliary clearance. Cold Spring Harb Perspect Biol 2017; 9 : a028241.

$7 \quad$ Wang SZ, Rosenberger CL, Espindola TM, et al. CCSP modulates airway dysfunction and host responses in an Ova-challenged mouse model. Am J Physiol Lung Cell Mol Physiol 2001; 281: L1303-L1311.

8 Tokita E, Tanabe T, Asano $\mathrm{K}$, et al. Club cell $10-\mathrm{kDa}$ protein attenuates airway mucus hypersecretion and inflammation. Eur Respir J 2014; 44: 1002-1010.

9 Laucho-Contreras ME, Polverino F, Gupta K, et al. Protective role for club cell secretory protein-16 (CC16) in the development of COPD. Eur Respir J 2015; 45: 1544-1556.

10 Miotto D, Ruggieri MP, Boschetto P, et al. Interleukin-13 and -4 expression in the central airways of smokers with chronic bronchitis. Eur Respir J 2003; 22: 602-608.

11 Alevy YG, Patel AC, Romero AG, et al. IL-13-induced airway mucus production is attenuated by MAPK13 inhibition. J Clin Invest 2012; 122: 4555-4568.

12 Schmid A, Sailland J, Novak L, et al. Modulation of Wnt signaling is essential for the differentiation of ciliated epithelial cells in human airways. FEBS Lett 2017; 591: 3493-3506.

13 Lomas DA, Silverman EK, Edwards LD, et al. Evaluation of serum CC-16 as a biomarker for COPD in the ECLIPSE cohort. Thorax 2008; 63: 1058-1063.

14 Vestbo J, Edwards LD, Scanlon PD, et al. Changes in forced expiratory volume in 1 second over time in COPD. N Engl J Med 2011; 365: 1184-1192.

15 Guerra S, Vasquez MM, Spangenberg A, et al. Club cell secretory protein in serum and bronchoalveolar lavage of patients with asthma. J Allergy Clin Immunol 2016; 138: 932-934. 